\title{
Acute fatal hemorrhage from previously undiagnosed cerebral arteriovenous malformations in children: a single-center experience
}

\author{
Coleman P. Riordan, BSc, ${ }^{1}$ Darren B. Orbach, MD, PhD, ${ }^{1,2}$ Edward R. Smith, MD, ${ }^{1}$ and \\ R. Michael Scott, MD' \\ Departments of ${ }^{1}$ Neurosurgery and ${ }^{2}$ Neurointerventional Radiology, Boston Children's Hospital, Boston, Massachusetts
}

OBJECTIVE The most significant adverse outcome of intracranial hemorrhage from an arteriovenous malformation $(A V M)$ is death. This study reviews a single-center experience with pediatric AVMs to quantify the incidence and characterize clinical and radiographic factors associated with sudden death from the hemorrhage of previously undiagnosed AVMs in children.

METHODS A single-center database review of the period from 2006 to 2017 identified all patients with a first-time intracranial hemorrhage from a previously undiagnosed AVM. Clinical and radiographic data were collected and compared between patients who survived to hospital discharge and those who died at presentation.

RESULTS A total of 57 patients (average age 10.8 years, range $0.1-19$ years) presented with first-time intracranial hemorrhage from a previously undiagnosed AVM during the study period. Of this group, $7 / 57$ (12\%) patients (average age 11.5 years, range $6-16$ years) suffered hemorrhages that led directly to their deaths. Compared to the cohort of patients who survived their hemorrhage, patients who died were 4 times more likely to have an AVM in the posterior fossa. No clear pattern of antecedent triggering activity (sports, trauma, etc.) was identified, and 3/7 (43\%) experienced cardiac arrest in the prehospital setting. Surviving patients were ultimately treated with resection of the AVM in $42 / 50(84 \%)$ of cases.

CONCLUSIONS Children who present with hemorrhage from a previously undiagnosed intracranial AVM had a $12 \%$ chance of sudden death in our single-institution series of pediatric cerebrovascular cases. Clinical triggers of hemorrhage are unpredictable, but subsequent radiographic evidence of a posterior fossa AVM was present in $57 \%$ of fatal cases, and all fatal cases were in locations with high risk of potential herniation. These data support a proactive, aggressive approach toward definitive treatment of AVMs in children.

https://thejns.org/doi/abs/10.3171/2018.3.PEDS1825

KEYWORDS arteriovenous malformation; hemorrhage; fatal; pediatric; cerebrovascular; outcome; vascular disorders

$\mathrm{T}$ HE management of intracranial arteriovenous malformations (AVMs) remains a topic of debate, with recent studies suggesting differing natural history between adult and pediatric populations. ${ }^{7,14}$ In general, most centers support a multidisciplinary approach to care, with a greater tendency to offer definitive treatment to children with AVMs (as opposed to observation in adults), given the long expected lifespan of a child and the largely favorable treatment outcomes across modalities. ${ }^{2,8,9} \mathrm{How}-$ ever, limited data exist to inform caregivers and families on the risk of sudden death from a previously undiagnosed intracranial AVM in children. In this study we sought to answer common clinical questions related to this topic, including incidence of death from first-time hemorrhage, identification of potential risk factors for hemorrhage, and characterization of any common radiographic findings present in these cases.

\section{Methods}

All work was approved by the institutional review board of Boston Children's Hospital (BCH). We identified 
TABLE 1. Summary of natural histories in 7 pediatric patients who died of ruptured undiagnosed AVMs between 2006 and 2017 at BCH

\begin{tabular}{ccclclll}
\hline $\begin{array}{c}\text { Case } \\
\text { No. }\end{array}$ & Sex & $\begin{array}{c}\text { Age at Death } \\
(\mathrm{yrs})^{*}\end{array}$ & AVM Location & $\begin{array}{c}\text { Time of } \\
\text { Bleed }\end{array}$ & $\begin{array}{c}\text { Activity Before } \\
\text { Bleed }\end{array}$ & Presentation & Past Medical History \\
\hline 1 & $\mathrm{~F}$ & 16.4 & Thalamus & AM & At school & Seizure, LOC & Asthma, anxiety \\
\hline 2 & $\mathrm{~F}$ & 13.4 & Rt temporal & AM & At school & HA, LOC w/ TBI & None \\
\hline 3 & $\mathrm{~F}$ & 6.8 & Cerebellum & PM & Birthday party & HA, LOC & None \\
\hline 4 & M & 11.6 & Lt thalamus & AM & At school & HA, seizure & $\begin{array}{c}\text { Febrile seizures \& HAs w/ no indica- } \\
\text { tion of structural abnormality }\end{array}$ \\
\hline 5 & M & 12.6 & Cerebellum & AM & Lacrosse practice & Cardiac arrest & Concussion 1 yr prior to hemorrhage \\
\hline 7 & M & 7.2 & Rt cerebellum & PM & Sleeping & HA, cardiac arrest & None \\
\hline
\end{tabular}

$\mathrm{HA}=$ headache; $\mathrm{LOC}=$ loss of consciousness; $\mathrm{TBI}$ = traumatic brain injury.

${ }^{*}$ Average age was $11.5 \pm 3.4$ years (mean $\pm \mathrm{SD}$ ).

patients who were admitted to the Emergency Department of $\mathrm{BCH}$ with a diagnosis of intracranial hemorrhage and AVM during the period from January 1, 2006, to September 30,2017 . This time period was chosen because it was concordant with the commencement of reliable institutional databases with cross-referencing capabilities. Patients were excluded from the analysis if there had been any neurosurgical or interventional treatment attempted prior to the hemorrhage, or if an AVM had been diagnosed prior to the admission hemorrhage, ensuring that all study patients were suffering first-time hemorrhages from previously undiagnosed AVMs. All radiographic studies were reviewed to confirm the diagnosis of AVM, supplemented with postmortem examinations when available. We reviewed all patient charts to determine demographic data, presenting symptoms, prehospital treatment, and clinical course. Radiographic studies were reviewed by a pediatric neuroradiologist (D.B.O.) for accuracy of diagnosis and key anatomical factors including size, location, and vascular supply.

\section{Results}

A total of 57 patients (average age 10.8 years, range 0.1-19 years) presented with first-time intracranial hemorrhage from a previously undiagnosed AVM during the study period. Of this group, 7/57 (12\%) patients (average age 11.5 years, range $6-16$ years) suffered hemorrhages that led directly to their death. Table 1 summarizes the characteristics of the patient population suffering sudden death, and Table 2 summarizes their therapeutic interventions, as well as the results of diagnostic examinations to confirm AVM presence. Two illustrative case histories are presented.

\section{Case 4}

This 11-year-old boy had a medical history significant for headaches and febrile seizures. He had undergone CT and MRI studies 7 years prior to admission that had been interpreted as normal, and which on retrospective analysis were similarly interpreted (Fig. 1). On the morning of admission, the patient complained of a headache to his school nurse, following which he had a generalized tonicclonic seizure. He presented to another hospital in status epilepticus, was treated with antiepileptic medication, and underwent CT scanning, which revealed a $2.2 \times 1.3-\mathrm{cm}$ left thalamic parenchymal hemorrhage with significant intraventricular extension and midline shift (Fig. 2A). An external ventricular drain (EVD) was placed and the child was then transferred to our institution. During transfer the patient continued to decline clinically, with decerebrate posturing.

Upon arrival at our hospital, a CTA study was performed, revealing a $3.4 \times 1.7 \times 0.9-\mathrm{cm}$ left thalamic AVM (Fig. 2B and C). The child continued to rapidly decline,

TABLE 2. Summary of emergency surgical intervention and diagnostic and postmortem examinations in 7 pediatric patients who died of ruptured undiagnosed AVMs between 2006 and 2017 at $\mathrm{BCH}$

\begin{tabular}{clll}
\hline Case No. & \multicolumn{1}{c}{ Surgical Intervention } & \multicolumn{1}{c}{ Diagnostic Exam } & Postmortem Exam \\
\hline 1 & Rt frontal craniotomy to evacuate intraventricular clot; rt frontal EVD & CTA & No \\
\hline 2 & $\begin{array}{c}\text { Rt frontal craniotomy to evacuate intraventricular clot; rt frontal EVD; embolization; rt } \\
\text { ventriculoperitoneal shunt }\end{array}$ & Angiogram/CTA/MRI & No \\
\hline 3 & No intervention attempted & CTA & Yes \\
\hline 4 & Rt frontal EVD & Angiogram/CTA/MRI & Unknown \\
\hline 5 & Suboccipital craniectomy \& C1 laminectomy to remove posterior fossa clot; rt occipital EVD & CTA & No \\
\hline 6 & Suboccipital craniectomy to decompress posterior fossa; rt frontal EVD & CTA & Yes \\
\hline 7 & Suboccipital craniectomy \& C1 laminectomy to evacuate clot; rt frontal EVD & CTA/MRI & Unknown \\
\hline
\end{tabular}



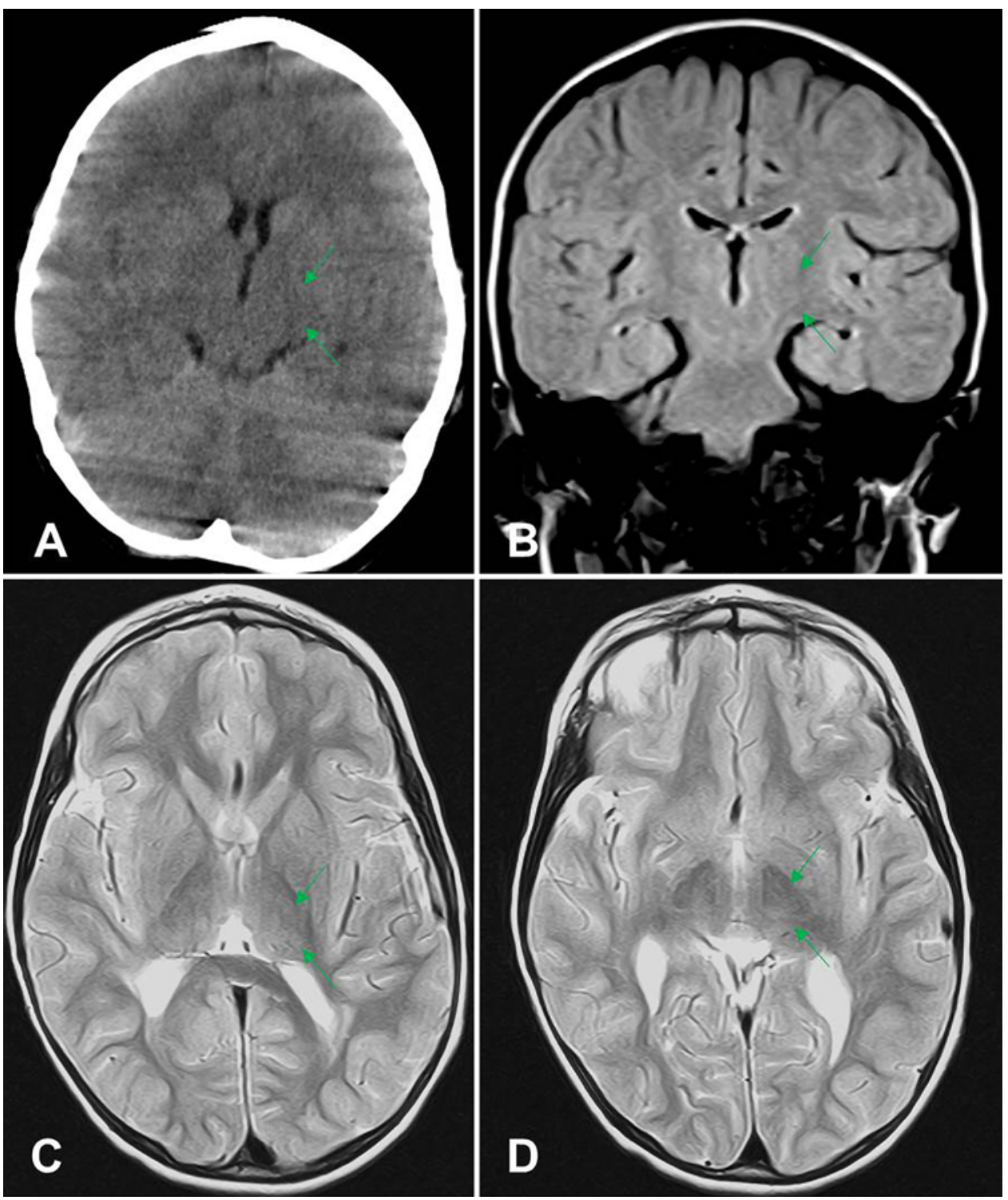

FIG. 1. Case 4. Imaging workup 7 years prior to the hemorrhage, performed for febrile seizures with headache. Axial noncontrast CT scan (A), coronal FLAIR MR scan (B), and axial T2-weighted MR scans ( $\mathbf{C}$ and D) all demonstrating normal brain parenchyma in the left thalamus (green arrows), with no flow voids, CT attenuation, or MR signal abnormality to suggest the presence of an underlying vascular lesion. Figure is available in color online only.

despite CSF drainage and hyperosmolar therapy, and demonstrated clinical evidence of herniation, including loss of brainstem reflexes and spontaneous respiration. MRI was performed for prognostic purposes, demonstrating extensive cortical and brainstem infarction (Fig. 2D-F). Care was withdrawn and the patient died.

\section{Case 7}

The patient was a 12-year-old boy with no significant past medical history. He was with his family in a rural setting when he suddenly grabbed his head and complained of a severe headache and photophobia shortly before collapsing. A family member noticed that the patient was cyanotic and not breathing and administered CPR until emergency medical services arrived. The patient was in- tubated, transported to another hospital, and then transferred to $\mathrm{BCH}$. On admission he underwent a CTA scan, which showed a 4.6-cm left cerebellar hemorrhage and a smaller right cerebellar hemorrhage with tonsillar herniation secondary to a ruptured AVM $(3.3 \times 1.2 \times 0.9 \mathrm{~cm})$ of the left cerebellum (Fig. 3).

Despite the poor prognosis, given the child's age and rapid evolution of symptoms, he was taken to the operating room for emergency clot evacuation and placement of a right frontal EVD in the hopes of salvaging function by rapid decompression. Following surgery, the patient exhibited no brainstem reflexes. MRI showed bilateral hemorrhage of the cerebellar hemispheres, with significant edema and brainstem injury. Brain death criteria were met and the child's organs were donated 3 days after the event. 

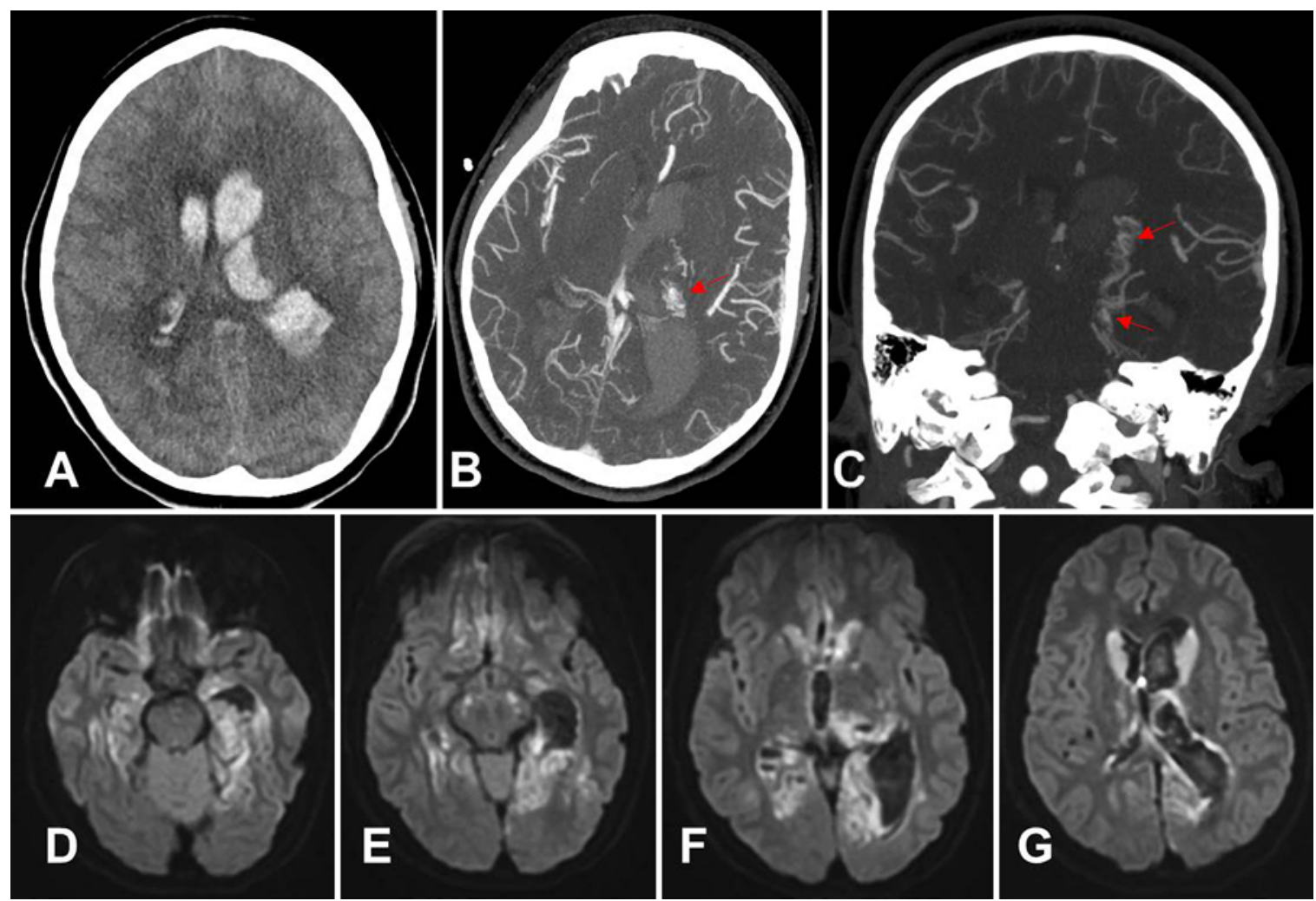

FIG. 2. Case 4. Imaging at the time of the acute hemorrhage. Noncontrast axial CT (A) demonstrates a left thalamic hemorrhage, with intraventricular extension and early midline shift. Axial (B) and coronal (C) CTA images demonstrate nidal vasculature consistent with AVM within the left thalamus (red arrows). Multiple axial diffusion-weighted MR images (D-F) demonstrate diffuse confluent and punctate foci of diffusion restriction in the cerebral peduncles, thalami, caudates, and temporal, frontal, and occipital lobes bilaterally. Figure is available in color online only.

\section{Additional Findings}

The 7 patients who died were slightly older than those who survived (11.5 years vs 10.7 years, not statistically significant). There was no ethnic or sex predilection noted between the two groups.

Past medical histories did not reveal significant predictive differences between the two groups. Only 1 of the patients who died (case 4, reported above) had a possibly relevant antecedent history of headaches and febrile seizures, which was evaluated with MRI 7 years prior to hemorrhage and reported as normal. In summary, there was no obvious set of clinical factors on history or physical examination that was predictive of the presence of the AVM.

Importantly, no clear immediate antecedent activity was identified that predicted subsequent fatal hemorrhage. There was no recent infection, trauma, or injury in any child, although 1 patient had a concussion the year prior to the hemorrhage. Activity at hemorrhage onset ranged from marked exertion (1 at lacrosse practice, 1 rope swinging), to sedate state (3 in school, 1 at a birthday party), to sleep (1). Moreover, despite the history of headache and seizure in previous years in 1 of the 7 patients, no buildup of any warning symptoms was reported by any patient or family in the fatal hemorrhage group.

Data on time interval from symptom onset to initial medical treatment was often approximate, due in part to the fact that patients were initially transported to other hospitals prior to transfer to $\mathrm{BCH}$, as well as due to inadequate documentation by hurried emergency medical service personnel in the midst of frantic patient triage. As a result, no reliable conclusions could be drawn about the relationship between symptom-treatment interval time and outcome.

All 7 patients who ultimately died had near-immediate loss of consciousness in the prehospital setting, a markedly higher rate than in the nonfatal hemorrhages (22\%). Of particular note, 3/7 (43\%) experienced cardiac arrest, in contrast to $0 \%$ in the nonfatal group. The presence of documented cardiac arrest was therefore strongly associated with a subsequent fatal outcome.

\section{Radiographic Findings}

Forty-six (92\%) of the 50 patients with nonfatal hemorrhages in this study had diagnostic preoperative arteriograms. In the remaining 4, the AVM diagnosis was made by either CTA (3) or MRI (1). Diagnostic examinations used for the fatal hemorrhage group are shown in Table 2 . Whereas all 7 patients underwent CTA, only 2 underwent angiography and 3 underwent MRI. Postmortem examinations were also performed in only 2 patients.

Radiographic studies were reviewed for all patients. No clear difference in size, arterial supply, or venous 

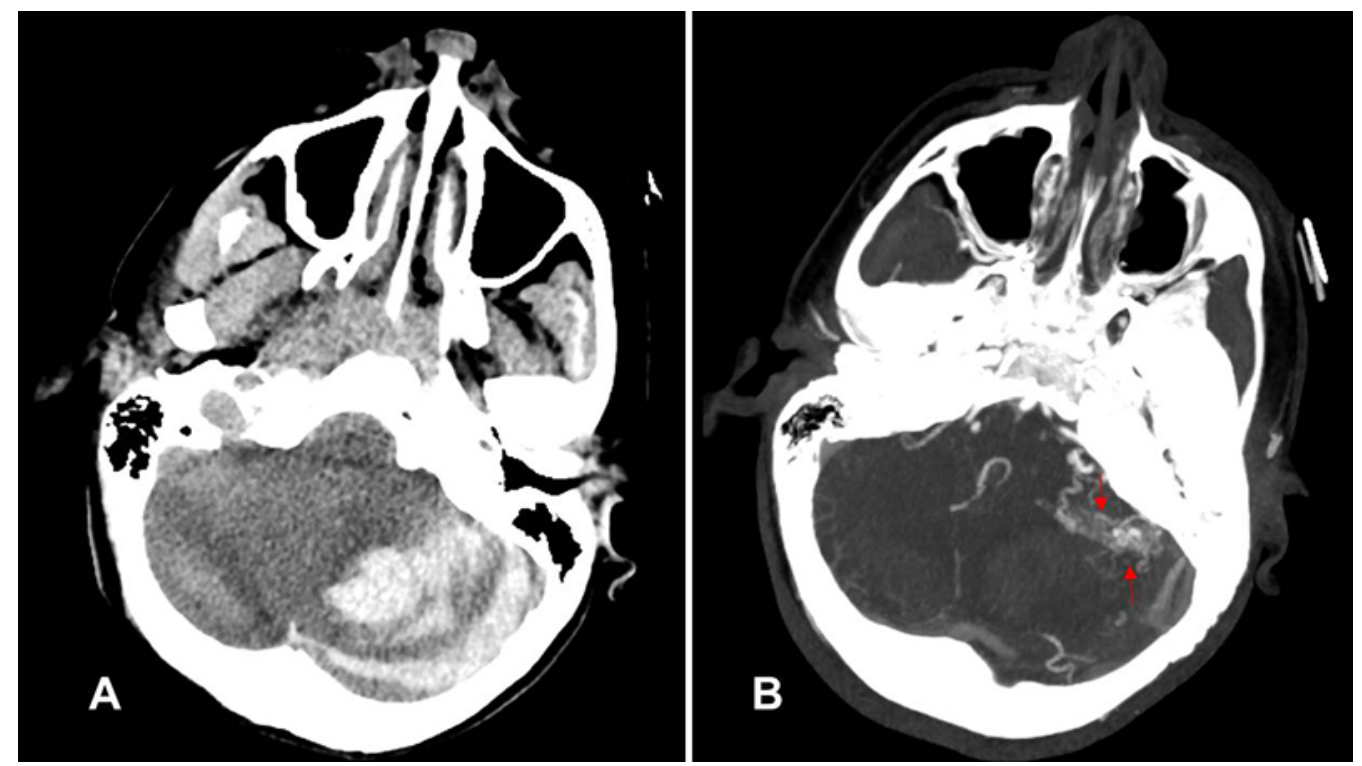

FIG. 3. Case 7. Axial noncontrast CT scan (A) demonstrating left cerebellar hemispheric parenchymal and subdural hemorrhage, and axial CTA (B) demonstrating AVM nidal vasculature within the left cerebellar hemisphere (red arrows). Figure is available in color online only.

anatomy could be discerned between the fatal and nonfatal hemorrhage groups. The one notable difference was the high prevalence of posterior fossa AVMs in the fatal hemorrhage group $(4 / 7[57 \%]$ in the sudden death cohort vs $7 / 50$ [14\%] in the nonfatal cohort). Children in the sudden death group of newly ruptured lesions therefore had a 4-fold greater likelihood of harboring a posterior fossa $\mathrm{AVM}$ as compared to the nonfatal cohort. Additionally, the 3 nonposterior fossa AVMs were either thalamic, adjacent to the midline ventricular system, or in the medial temporal lobe adjacent to the brainstem; thus, there was no case of fatal initial hemorrhage involving a true lobar hemispheric AVM.

\section{Discussion}

Most of the current literature on intracranial AVMs focuses on the adult population, with limited data regarding pediatric patients. Clinical decisions based on studies of adult populations may not be directly applicable to children, because children with symptomatic AVMs are thought to have a higher likelihood of bleeding (80\%) than adults $(50 \%-65 \%)$ and spontaneous hemorrhage rates may differ, as well as responses to treatment.,3,13 Previous studies have suggested that death from an initial bleed of an AVM may be anywhere from $4 \%$ to $29 \%{ }^{4,5,16}$ Here we present a single-center study focused specifically on pediatric patients with AVM who harbored previously undiagnosed lesions and who presented with first-time hemorrhage. Our purpose was to ascertain mortality rates and discern any common clinical or radiographic attributes, to better inform clinicians caring for this population.

\section{General Prevalence of Sudden Death From First-Time Hemorrhage of AVM}

Our 12-year review identified 57 pediatric patients (ages 0.1-19 years) who experienced a radiographically confirmed first-time hemorrhage from a previously undiagnosed intracranial AVM. Of these 57 patients, 7 died as a result of the initial hemorrhage, yielding a first-time hemorrhage mortality rate of $12 \%$. This finding is within the range of other reported series of AVM hemorrhage-related mortality, but is higher than reported mortality rates for pediatric intracranial hemorrhage from any cause, and is unique in its specificity for focusing on the population of pediatric patients and those uniquely characterized as experiencing first-time bleeds from undiagnosed AVMs. ${ }^{6,12,15,17}$ As such, this study may provide a useful benchmark for clinicians in calculating risk of death from initial hemorrhage in children.

\section{Clinical and Radiographic Associations}

There was no significant age, ethnic, or sex predilection noted between the patients who died from the initial hemorrhage and those who survived. Past medical history reviews were also unable to predict the presence or risk of AVM hemorrhage. Only 1 patient who died was noted to have any medical history that could possibly be consistent with an intracranial vascular malformation-case 4-who had a history notable for headaches and febrile seizures. However, cranial MRI and CT scans 7 years prior to the rupture showed no evidence of any vascular lesion (Fig. 1). Despite these initially negative studies, CTA performed at the time of presentation clearly displayed a defined AVM nidus in the left thalamus (Fig. 2). In summary, we were unable to identify any useful demographic or clinical history data that could help to predict the presence of a subsequently fatal AVM.

A common question in our practice centers on the idea of an inciting activity that might serve as a "trigger" for AVM hemorrhage. In particular, families will often ask 
about the risk of sports, amusement park rides, or trauma. Previous literature has suggested that these activities do not seem to correlate with increased rates of AVM hemorrhage. ${ }^{17}$ In our 7 acutely fatal cases, 6 hemorrhages occurred during the day and 1 while the patient was sleeping. No clear immediate antecedent activity was identified that predicted subsequent fatal hemorrhage. There was no recent infection, trauma, or injury in any child, although 1 patient had a concussion the year prior to hemorrhage. Activity ranged from marked exertion (1 at lacrosse practice, 1 rope swinging), to a relatively sedate state (3 at school, 1 at a birthday party), to sleep (1). Moreover, despite the history of headache and seizure for years in 1 patient, no buildup of warning symptoms occurred in any patient. In these children harboring an undiagnosed AVM, there appeared to be no specific trigger that may have led to the catastrophic fatal hemorrhage.

Based on location alone, there were notable differences between the 7 patients who died of the initial hemorrhage and the 50 who survived. It has been reported that AVMs of the cerebellum and brainstem are at a higher risk of hemorrhage compared to supratentorial AVMs. ${ }^{1,5}$ In our series, $46 / 57(80.7 \%)$ of the AVMs were supratentorial, with the majority of these $(21 / 46 ; 45.7 \%)$ occurring in temporal or parietotemporal areas. However, of the 11 hemorrhages from infratentorial AVMs, 4 (36.4\%) led to fatal outcomes. Although we cannot report a higher incidence of hemorrhage from infratentorial bleeds, we do find a significantly higher rate of mortality from infratentorial AVM hemorrhages $(4 / 11,36.4 \%)$ compared to supratentorial $(3 / 46,6.5 \%)$. Although infratentorial AVMs make up only about $25 \%$ of all surgically treated AVMs, this location has been previously identified as a significant risk factor for symptomatic bleeding. ${ }^{5,10}$ It is notable that all our patients with fatal hemorrhage had AVMs in locations presenting high risk of potential central, uncal, or tonsillar herniation. Taken together, the data from this study suggest that anatomical location of an AVM in the thalamus or posterior fossa increases the risk of death from firsttime hemorrhage by 4 -fold in pediatric patients.

\section{Limitations of the Study}

This study is limited by its retrospective nature and the relatively small cohort of patients, despite coming from a large-volume center. This study was not meant to be epidemiological in nature, defining a specific mortality rate, but rather it was meant to demonstrate the potential for a serious outcome of AVM hemorrhages in the pediatric population and to present the experience of a single tertiary pediatric hospital in this regard. In addition, despite being a review that incorporates modern imaging techniques, there is a lack of consistent angiographic and pathological data due to the emergency nature of the fatal cases and the unwillingness of some of the families to permit an autopsy. Last, also due to the urgency of the cases, imprecision of emergency responder notes, and the tendency for patients to be transported to other hospitals prior to transfer to $\mathrm{BCH}$, information on time between onset of symptoms, arrival at the first hospital, and admission to $\mathrm{BCH}$ was difficult to obtain and to define precisely. It is our hope that the subgroup analysis provided by this work will help to further inform caregivers and families about salient risks of pediatric AVMs.

\section{Recommendations}

The malignant natural history of pediatric intracranial AVMs, with high lifetime rates of hemorrhage in children and a major risk of death with each bleeding event, supports an aggressive stance toward providing definitive treatment whenever possible. ${ }^{4,16}$ The generally low morbidity of multimodal therapies for pediatric AVMs, coupled with excellent, durable long-term cure rates, suggest that treatment should be offered whenever feasible. ${ }^{4,8,11,16}$

\section{Conclusions}

In this consecutive series of pediatric patients with AVM, children who presented with hemorrhage from a previously undiagnosed intracranial AVM had a $12 \%$ chance of sudden death. Clinical triggers of hemorrhage were unpredictable, but radiographic evidence of a posterior fossa AVM was present in 57\% of fatal cases, and all fatal cases were in locations with high risk of potential herniation. These data support a proactive, aggressive approach toward definitive treatment of AVMs in children.

\section{Acknowledgments}

We would like to acknowledge The Lucas Warner Fund, The Fellows Fund, and The Oxley Research Fund for supporting this project.

\section{References}

1. Baskaya MK, Jea A, Heros RC, Javahary R, Sultan A: Cerebral arteriovenous malformations. Clin Neurosurg 53:114144, 2006

2. Börcek AO, Emmez H, Akkan KM, Öcal Ö, Kurt G, Aykol $S$, et al: Gamma Knife radiosurgery for arteriovenous malformations in pediatric patients. Childs Nerv Syst 30:14851492,2014

3. Cognard C, Spelle L, Pierot L: Pial arteriovenous malformations, in Forsting M, Wanke I (eds): Intracranial Vascular Malformations and Aneurysms, ed 2. Berlin: Springer, 2008, pp 51-74

4. Ding D, Starke RM, Kano H, Mathieu D, Huang PP, Feliciano C, et al: International multicenter cohort study of pediatric brain arteriovenous malformations. Part 1: Predictors of hemorrhagic presentation. J Neurosurg Pediatr 19:127-135, 2017

5. Ellis MJ, Armstrong D, Vachhrajani S, Kulkarni AV, Dirks PB, Drake JM, et al: Angioarchitectural features associated with hemorrhagic presentation in pediatric cerebral arteriovenous malformations. J Neurointerv Surg 5:191-195, 2013

6. Fox CK, Johnston SC, Sidney S, Fullerton HJ: High critical care usage due to pediatric stroke: results of a populationbased study. Neurology 79:420-427, 2012

7. Gross BA, Scott RM, Smith ER: Management of brain arteriovenous malformations. Lancet 383:1635, 2014

8. Gross BA, Storey A, Orbach DB, Scott RM, Smith ER: Microsurgical treatment of arteriovenous malformations in pediatric patients: the Boston Children's Hospital experience. J Neurosurg Pediatr 15:71-77, 2015

9. Ladner TR, Mahdi J, Attia A, Froehler MT, Le TM, Lorinc AN, et al: A multispecialty pediatric neurovascular conference: a model for interdisciplinary management of complex disease. Pediatr Neurol 52:165-173, 2015 
10. Lawton MT, Rutledge WC, Kim H, Stapf C, Whitehead KJ, Li DY, et al: Brain arteriovenous malformations. Nat Rev Dis Primers 1:15008, 2015

11. Lin N, Smith ER, Scott RM, Orbach DB: Safety of neuroangiography and embolization in children: complication analysis of 697 consecutive procedures in 394 patients. J Neurosurg Pediatr 16:432-438, 2015

12. Liu J, Wang D, Lei C, Xiong Y, Yuan R, Hao Z, et al: Etiology, clinical characteristics and prognosis of spontaneous intracerebral hemorrhage in children: a prospective cohort study in China. J Neurol Sci 358:367-370, 2015

13. Maher CO, Scott RM: Linear vein-based arteriovenous malformations in children. J Neurosurg Pediatr 4:12-16, 2009

14. Mohr JP, Parides MK, Stapf C, Moquete E, Moy CS, Overbey JR, et al: Medical management with or without interventional therapy for unruptured brain arteriovenous malformations (ARUBA): a multicentre, non-blinded, randomised trial. Lancet 383:614-621, 2014

15. Reitz M, von Spreckelsen N, Vettorazzi E, Burkhardt T, Grzyska U, Fiehler J, et al: Angioarchitectural risk factors for hemorrhage and clinical long-term outcome in pediatric patients with cerebral arteriovenous malformations. World Neurosurg 89:540-551, 2016

16. Roach ES, Golomb MR, Adams R, Biller J, Daniels S, Deveber $\mathrm{G}$, et al: Management of stroke in infants and children: a scientific statement from a Special Writing Group of the
American Heart Association Stroke Council and the Council on Cardiovascular Disease in the Young. Stroke 39:26442691, 2008

17. Singhal NS, Hills NK, Sidney S, Fullerton HJ: Role of trauma and infection in childhood hemorrhagic stroke due to vascular lesions. Neurology 81:581-584, 2013

\section{Disclosures}

The authors report no conflict of interest concerning the materials or methods used in this study or the findings specified in this paper.

\section{Author Contributions}

Conception and design: Scott. Acquisition of data: all authors. Analysis and interpretation of data: all authors. Drafting the article: Scott, Riordan, Smith. Critically revising the article: all authors. Reviewed submitted version of manuscript: all authors. Approved the final version of the manuscript on behalf of all authors: Scott. Administrative/technical/material support: Orbach, Smith. Study supervision: Scott.

\section{Correspondence}

R. Michael Scott: Boston Children's Hospital, Boston, MA. michael.scott@childrens.harvard.edu. 